\title{
Two-stage genome-wide association study for the identification of causal variants underlying hoof disorders in cattle
}

\author{
T. Suchocki, ${ }^{1,2 *} \odot$ Ch. Egger-Danner, ${ }^{3} \odot$ H. Schwarzenbacher, ${ }^{3}$ and J. Szyda ${ }^{1,2} \odot$ \\ ${ }^{1}$ Biostatistics group, Department of Genetics, Wroclaw University of Environmental and Life Sciences, Kozuchowska 7, 51-631 Wroclaw, Poland \\ ${ }^{2}$ National Research Institute of Animal Production, Krakowska 1, 32-083 Balice, Poland \\ ${ }^{3}$ ZuchtData EDV-Dienstleistungen GmbH, Dresdner Straße 89/19, 1200 Vienna, Austria
}

\section{ABSTRACT}

Feet and legs disorders influence dairy cattle breeding by their effect on animal welfare, economic losses due to lower production and fertility, costs of treatment, and problems with herd management. In our study, we estimated heritabilities and performed a 2-step GWAS for 3 traits describing hoof health: hoof health status defined by a veterinarian (HSV), hoof health status defined by a claw trimmer (HSC), and the total number of hoof disorders (NHD), scored in 1,998 Fleckvieh and 979 Braunvieh cows. The individuals were genotyped with a high-density (HD) panel consisting of 76,934 SNP. For significant genomic regions, the SNP information was enhanced by SNP imputed from the wholegenome sequence of Fleckvieh and Braunvieh bulls from the 1000 Bulls Genome project. The heritabilities were estimated to be 0.035 for HSV, 0.249 for HSC, and 0.279 for NHD. Based on the first-stage GWAS with SNP from the HD panel, 7 significant genomic regions on 6 chromosomes were defined: (1) 120 SNP spanning 15,522 bp on BTA1, including the TOPBP1 gene; (2) 4,139 SNP spanning 1,426,046 bp on BTA7, including the RIOK2 and RGMB genes; (3) $167 \mathrm{SNP}$ spanning 167,352 bp on BTA13, including the C13H20orf194 gene; (4) 2 regions on BTA14, one harboring 1,071 SNP spanning 380,024 bp, including RRM2B and NCALD, and the other comprising 632 SNP spanning 385,111 bp, including STK3; (5) 328 SNP on BTA15, spanning 235,567 bp between FAM168A and PLEKHB1; and (6) 1,549 SNP on BTA22, spanning 596,101 bp in the neighborhood of PTPRG. Then, we conducted a second-stage GWAS based on SNP from whole-genome sequences within the significant regions obtained in the first stage of the analysis. For HSV, the highest additive effect was estimated for 23 SNP located within a region on BTA15, close to FAM168A, corresponding to

Received September 4, 2019.

Accepted December 11, 2019.

*Corresponding author: tomasz.suchocki@upwr.edu.pl a predicted gene sequence. For HSC, the highest additive effect was attributed to 44 SNP located within a region of BTA22 corresponding to 4 predicted gene sequences, with rs135082893 within a sequence encoding a microRNA. Another potential causal mutation for HSC was rs134142607 on BTA13, within the exon of C13H20orf194. For NHD, 33 SNP with the highest estimated effect were located on BTA7 within a region of a predicted gene positioned between RIOK2 and $R G M B$. On BTA14, all significant SNP were located in introns of $S T K 3$, which is responsible for the "abnormal gait" phenotype in mice.

Key words: Braunvieh, hoof disorders, Fleckvieh, genome-wide association study (GWAS), imputation to sequence

\section{INTRODUCTION}

Feet and legs disorders influence dairy cattle breeding in various aspects. The most important is their effect on animal welfare, followed by economic losses due to lower production levels and problems with fertility, and finally by costs of treatment. Another negative effect related to these disorders is associated problems with herd management because of the incidence of involuntary culling of sick cows. Heritabilities of feet and legs disorders vary between 0.005 (Ring et al., 2018) and 0.34 (Swalve et al., 2008). Therefore, it is possible (and important) to identify genes that are responsible for variation in animals' susceptibility to feet and legs disorders. This will enable establishing genetic programs aimed at reducing the incidence of feet and legs disorders.

A key problem in the analysis of feet and legs disorders is the lack of routine recording of those traits in most countries and thus the shortage of a sufficient number of phenotypes to estimate genetic parameters and conduct GWAS. Some such studies, all relatively recent, exist (for a comprehensive review, see Heringstad et al., 2018). Chapinal et al. (2013), Ødegård et al. (2013), van der Spek et al. (2013), Weber et al. (2013) 
Pérez-Cabal and Charfeddine (2015), Malchiodi et al. (2017) and Croué et al. $(2017,2019)$ estimated variance components for various traits related to feet and legs disorders. Buitenhuis et al. (2007), Swalve et al. (2014), and van der Spek et al. (2015) detected genomic regions responsible for variation in the disorders. In the latter context, the recent study of Wu et al. (2016) was especially valuable, because the authors used SNPs imputed from a 50K chip to whole-genome sequence (WGS) variants to estimate additive effects of the polymorphisms on the susceptibility to feet and legs disorders. However, despite a large sample size of 10,751 bulls representing 3 breeds and a very large number of SNPs $(22,751,039)$, the study identified a small number of significant variants and found no overlap between the 3 analyzed breeds.

In our study, we followed Wu et al. (2016) in that we combined SNP chip and WGS information in a GWAS for hoof disorders to identify candidate genes harboring causal mutations in Braunvieh and Fleckvieh. However, to minimize the type I and type II error risk due to testing millions of single SNP-phenotype associations in a mid-sized sample, we used a 2-stage GWAS procedure.

\section{MATERIALS AND METHODS}

\section{Data Set}

The analyzed data set comprised records of hoof disorders scored on Austrian Braunvieh and Fleckvieh cows over several parities (between 1 and 13) within the frame of the Efficient Cow project (http://en.zar .at/Projects/Efficient-Cow.html). For each cow, 3 lameness-related traits collected until 100 DIM were considered: hoof health status defined by a veterinarian (HSV; binary), hoof health status defined by a claw trimmer (HSC; binary), and total number of hoof disorders (NHD; discrete values between 0 and 5 ). The incidence of hoof disorders as defined by the veterinarian varied between $3.55 \%$ in parity 1 and $7.82 \%$ in parities $>4$ (Figure 1). The incidence of hoof disorders defined by a claw trimmer was much higher and varied between $38.32 \%$ in parity 1 and $60.03 \%$ in parities $>4$ (Figure 2). In each parity, the largest fraction of cows were healthy with no hoof disorders, and the maximum number of 584 diagnoses was recorded for parities $>4$ (Figure 3). The number of cows with records varied between 2,977 for HSV (1,998 Fleckvieh and 979 Braunvieh) and 1,513 for HSC and NHD (1,064 Fleckvieh and 449 Braunvieh). Interestingly, after 100 DIM, no increase in disorder incidence was observed - prolonging trait recording until 300 DIM resulted in only a few additional diagnoses of hoof problems (Table 1). Therefore, only traits recorded until 100 DIM were ana-

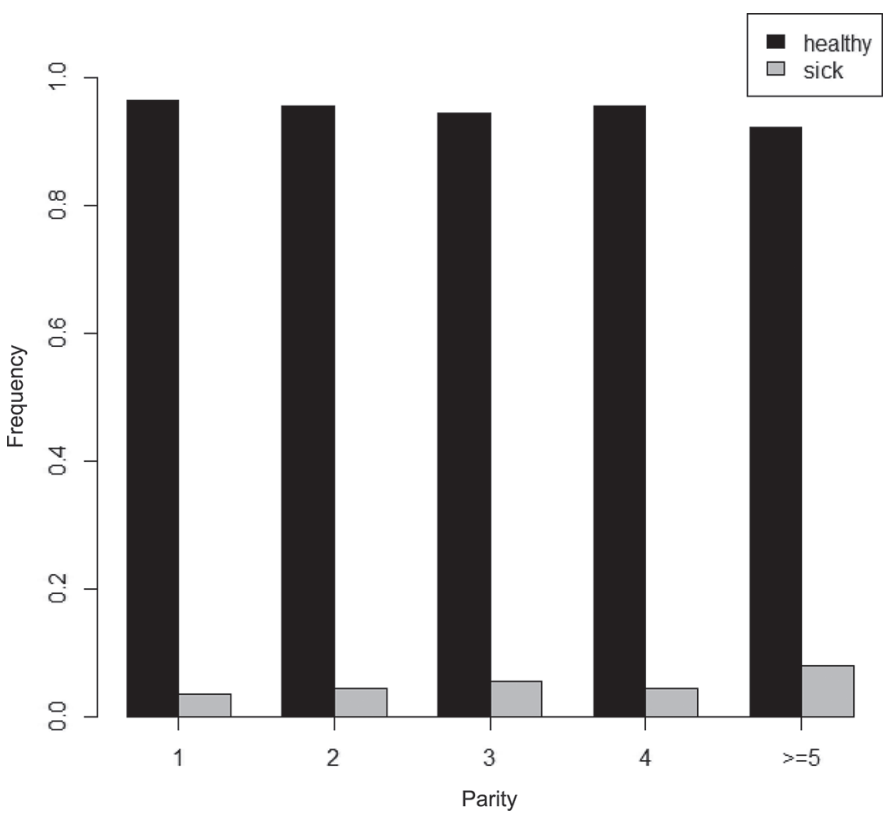

Figure 1. The incidence of hoof health status defined by a veterinarian (HSV).

lyzed. The number of recorded parities per cow ranged between 1 and 8. Each cow was genotyped with the GeneSeek Genomic Profiler HD panel (Neogen Genomics, Lincoln, NE) consisting of 76,934 SNPs. The SNP preselection criteria comprised a minor allele frequency (MAF) of at least 0.01 and technical quality of genotyping expressed by a minimum call rate of $99 \%$. After editing, 74,762 SNPs remained for further analysis, exhibiting an average MAF of 0.31 and an average call rate of $99.48 \%$.

\section{Variance Components}

For NHD, variance components were estimated using the following linear mixed model:

$$
\mathbf{y}=\mathbf{X} \boldsymbol{\beta}+\mathbf{Z}_{\mathbf{u}} \mathbf{u}+\mathbf{Z}_{\mathbf{v}} \mathbf{v}+\mathbf{Z}_{\mathrm{p}} \mathbf{p}+\varepsilon,
$$

where $\mathbf{y}$ is a vector of all records for NHD, including multiple parities; $\boldsymbol{\beta}$ is a vector of fixed effects comprising a general mean, breed, parity (from 1 to 4 and $>4$ ), calving year-season [composed of years 2012-2015 and season 1 (October-March) and 2 (April-September)]; percent of non-Holstein-Friesian genes (varying between 0 and $49 \%)$ and hoof status recording code $(0=$ unknown, $1=$ officer, $2=$ electronic via a vet, $3=$ cow owner); $\mathbf{u}$ is a random additive polygenic effect of a cow; $\mathbf{p}$ is a random permanent environmental effect; $\mathbf{v}$ is a random veterinarian effect, and $\varepsilon$ is a vector of random residuals. It was assumed that $\mathbf{u} \sim N\left(0, \mathbf{A} \sigma_{u}^{2}\right)$, 
Table 1. Descriptive statistics of traits

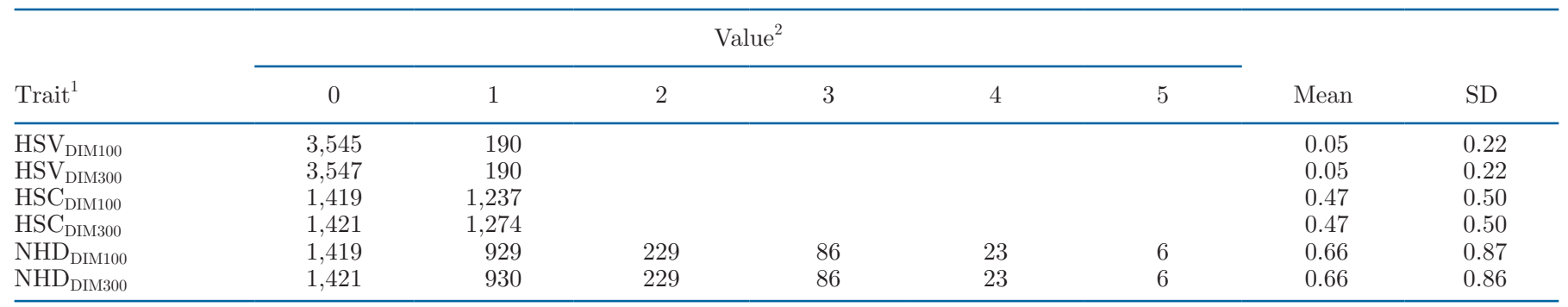

${ }^{1} \mathrm{HSV}=$ hoof health status defined by a veterinarian; HSC = hoof health status defined by a claw trimmer; NHD = total number of hoof disorders; DIM100 = traits scored until 100 DIM; DIM300 = traits scored until 300 DIM.

${ }^{2} \mathrm{HSV}$ and HSC were binary traits with values of 0 and 1 ; NHD had discrete values from 0 to 5.

$\mathbf{v} \sim N\left(0, \mathbf{I}_{\mathbf{v}} \sigma_{v}^{2}\right), \quad \mathbf{p} \sim N\left(0, \mathbf{I}_{\mathbf{p}} \sigma_{p}^{2}\right)$, and $\varepsilon \sim N\left(0, \mathbf{I}_{\varepsilon} \sigma_{\varepsilon}^{2}\right)$, where $\mathbf{A}$ is an additive relationship matrix among individuals calculated based on pedigree; $\sigma_{u}^{2}, \sigma_{v}^{2}, \sigma_{p}^{2}$, and $\sigma_{\varepsilon}^{2}$ are variances for additive polygenic, veterinarian, permanent environmental, and residual effects, respectively; $\mathbf{I}_{v}, \mathbf{I}_{\mathrm{p}}$, and $\mathbf{I}_{\mathbf{e}}$ are identity matrices with dimension equal to vectors $\mathbf{v}, \mathbf{p}$, and $\mathbf{e}$, respectively; and $\mathbf{X}, \mathbf{Z}_{\mathbf{u}}, \mathbf{Z}_{\mathbf{v}}$, and $\mathbf{Z}_{\mathbf{p}}$ are design matrices for $\boldsymbol{\beta}, u, v$, and $p$, respectively.

For the binary traits HSV and HSC, variance components were estimated using the following logistic model:

$$
\operatorname{logit}(\mathbf{p})=\mathbf{X} \boldsymbol{\beta}+\mathbf{Z}_{\mathbf{u}} u+\mathbf{Z}_{\mathbf{v}} v+\mathbf{Z}_{\mathbf{p}} p+\varepsilon,
$$

with $\mathbf{p}$ representing the probability of hoof disease diagnosis and elements on the right-hand side of model
[2] being the same as in model [1] and depending on a trait $v$ denoting a veterinarian or a claw trimmer effect. Effects of both models were estimated using the ASReml v3.0 software (Butler et al., 2009).

\section{First-Stage GWAS with SNPs from the HD Panel}

The additive effects of SNPs from the HD panel were estimated by applying the model for NHD:

$$
\mathbf{y}=\mathbf{X} \boldsymbol{\beta}+\mathbf{X}_{\mathrm{g}} g+\mathbf{Z}_{\mathbf{u}} u+\mathbf{Z}_{\mathbf{v}} v+\mathbf{Z}_{\mathbf{p}} p+\varepsilon,
$$

and for HSV and HSC:

$$
\operatorname{logit}(\mathbf{p})=\mathbf{X} \boldsymbol{\beta}+\mathbf{X}_{\mathbf{g}} g+\mathbf{Z}_{\mathbf{u}} u+\mathbf{Z}_{\mathbf{v}} v+\mathbf{Z}_{\mathbf{p}} p+\varepsilon,[4]
$$

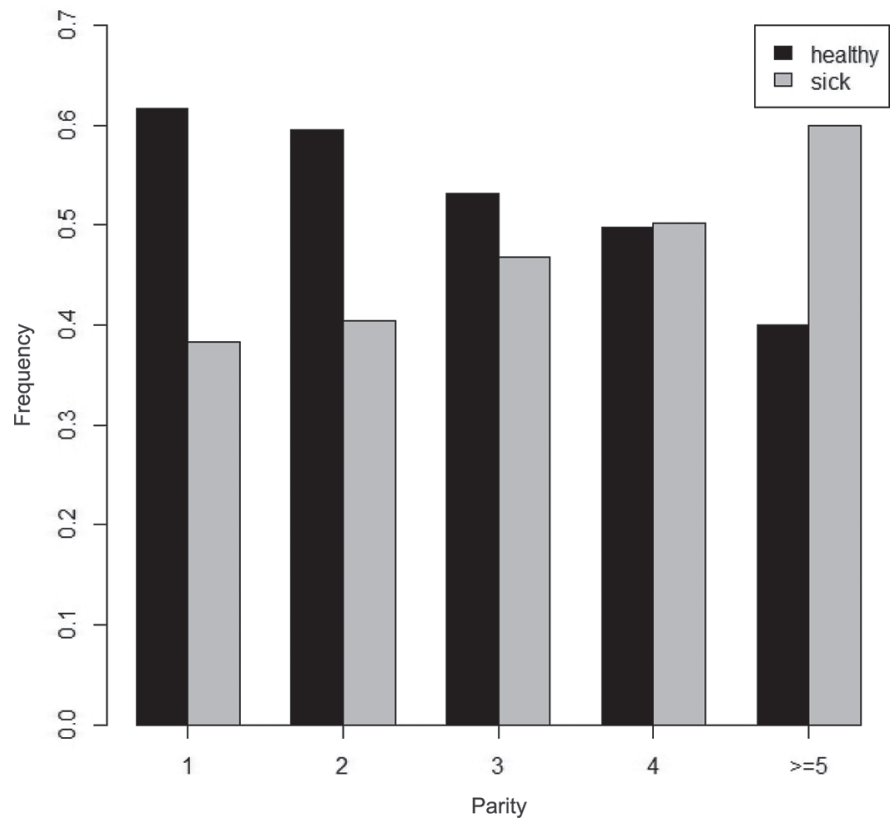

Figure 2. The incidence of hoof health status defined by a claw trimmer (HSC).

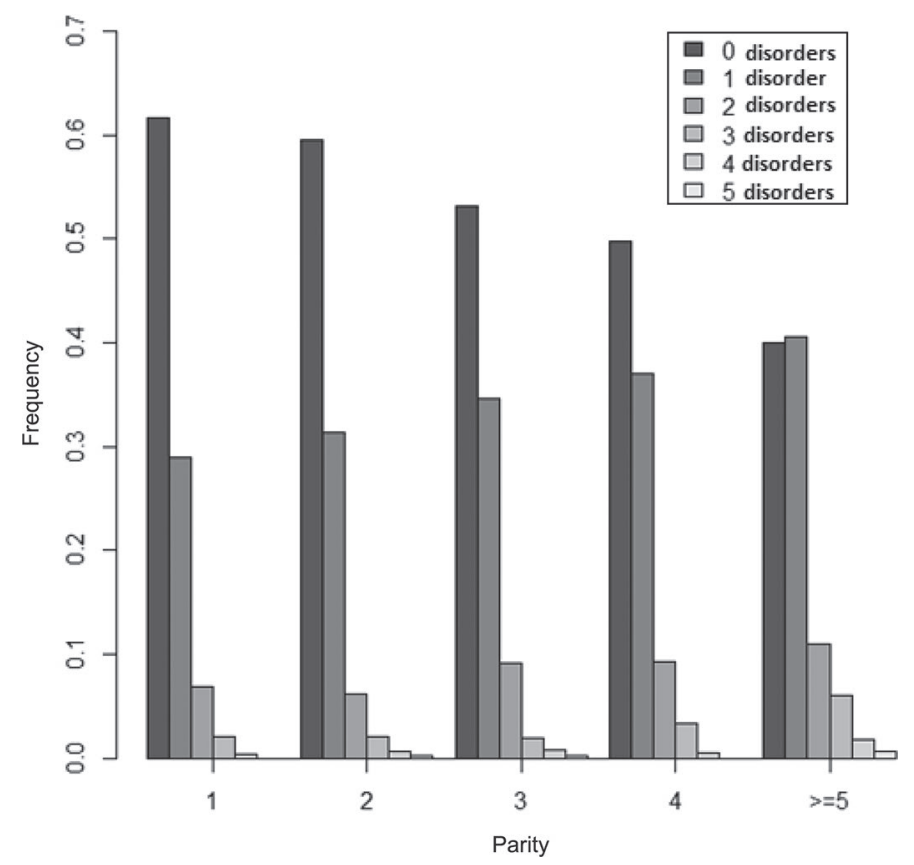

Figure 3. The incidence of hoof disorders (NHD). 
which complemented the right-hand side of equations [1] and [2] by $\mathbf{X}_{\mathrm{g}}$ g, where $\mathbf{X}_{\mathrm{g}}$ is a design vector for SNP genotypes, parameterized as $-1,0$, or 1 for a homozygous, heterozygous, and an alternative homozygous SNP genotype, respectively, and $g$ represents an additive fixed SNP effect. The procedure was repeated for each SNP separately, with the variance components corresponding to random effects assumed as known and set to the estimates obtained in models [1] and [2]. To test the hypotheses $\left(\mathrm{H}_{0}: g=0\right.$ vs. $\left.\mathrm{H}_{1}: g \neq 0\right)$, the Wald test, $W=\hat{g} / \sigma_{\hat{g}}$, was used, where $\sigma_{\hat{g}}$ is the standard error of the estimated SNP effect $\hat{g}$. Under $\mathrm{H}_{0}$, this statistic followed the standard normal distribution. The multiple testing correction via Bonferroni's approach (Dunnett, 1955) does not account for the intercorrelation between tests, which here arises through linkage disequilibrium (LD) between SNPs, and therefore does not guarantee a predefined experiment-wise type I error rate. Therefore, 2 methods for estimating the effective number of tests $\left(m_{e}^{1}\right.$ and $\left.m_{e}^{2}\right)$, accounting for the LD between SNPs, were applied. Let $m$ be the number of tests performed for each SNP and $\lambda_{i}(i=1, \ldots, m)$ be the eigenvalues of a pairwise LD matrix between SNPs. The first estimator is given by

$$
m_{e}^{1}=\sum_{i=1}^{m}\left[I\left(\lambda_{i} \geq 1\right)+\left(\lambda_{i}-\left|\lambda_{i}\right|\right)\right]
$$

where $I(\cdot)$ is an indicator function and $[\cdot]$ is a floor function ( $\mathrm{Li}$ and $\mathrm{Ji}, 2005$ ). The second estimator, given by

$$
m_{e}^{2}=m-\sum_{i=1}^{m} I\left(\lambda_{i}>1\right)\left(\lambda_{i}-1\right)
$$

was proposed by Li et al. (2012); $m_{e}^{1}$ and $m_{e}^{2}$ were used for multiple testing correction of nominal $P$-values in place of the total number of tests $(m)$ in the Bonferroni procedure. The main advantage of using $m_{e}^{1}$ and $m_{e}^{2}$ is that they account for the intercorrelation between tests and thus are lower than $m$, resulting in less conservative correction of the nominal significance.

\section{Second-Stage GWAS with SNPs from WGS}

Whole-genome DNA sequences of Braunvieh (50 individuals) and of Fleckvieh (30 individuals), available through the 1000 Bulls Genome Project (Hayes and Daetwyler, 2019), were used for the imputation of SNPs of phenotyped individuals within the significant genomic regions defined by the first-stage GWAS. Im- putation was carried out separately for each breed using Beagle 4 software (Browning and Browning 2007). A significant genomic region was defined based on the location of the most significant SNP from the HD panel and positions of the closest downstream and upstream genes. It comprised SNPs from the HD panel and imputed SNPs from WGS with MAF $>0$ (Table 2). Then, the additive effect of each SNP within the significant region was estimated using the same models as used for SNPs from the HD panel.

\section{RESULTS}

The lowest heritability of 0.035 was estimated for hoof health status defined by a veterinarian (HSV). For the remaining traits, similar heritabilities of 0.249 for hoof health status defined by a claw trimmer (HSC) and 0.279 for the total number of hoof disorders (NHD) were estimated (Table 3 ).

The estimated number of independent tests was lowest for $m_{e}^{1}(69,421)$ and 71,233 for $m_{e}^{2}$, which resulted in reductions of, respectively, $7.1 \%$ and $4.7 \%$ compared with the number of tests in Bonferroni correction $(74,762)$. Practically, these reductions did not affect the selection of significant SNPs in our GWAS models. Thus, the significance threshold after Bonferroni correction was set to 0.05 . The GWAS detected 2 significant SNP for HSV located on BTA14 and BTA15; 3 significant SNP for HSC located on BTA1, BTA13, and BTA22; and 2 significant SNP for NHD located on BTA7 and BTA14 (Figures 4, 5, and 6, Table 4). Based on those locations, significant genomic regions were examined in detail using SNP imputed from WGS.

For HSV, the significant region defined on BTA14 spanned 380,024 bp between rs135667758 located in the intron of RRM2B and rs381281819 in the intron of NCALD (Figure 7, Table 2). Among the 1,071 SNP located within this region, the most significant group comprised 70 neighboring SNP spanning 11,049 bp. Because of complete LD between them, no new information was gained by WGS because the region contained the most significant SNP identified in the first-stage GWAS with the HD panel. All polymorphisms were in an intergenic region downstream of $N C A L D$, which additionally corresponded to an intron of a computationally predicted gene (Table 5). The second significant region for HSV was at $235,567 \mathrm{bp}$ on BTA15 between rs110476174 and rs385044070 (Figure 7, Table 2). Among the 328 SNP in this region, the highest effect (and thus significance) in the stage-2 GWAS was estimated for 23 SNP in complete $\mathrm{LD}$, spanning $8,055 \mathrm{bp}$ of an intergenic region between FAM168A and PLEKHB1, a sequence that corresponds to a computationally predicted gene (Table 5). As for BTA14, the BTA15 SNP from WGS 
Table 2. Significant regions from the high-density $(\mathrm{HD})$ panel

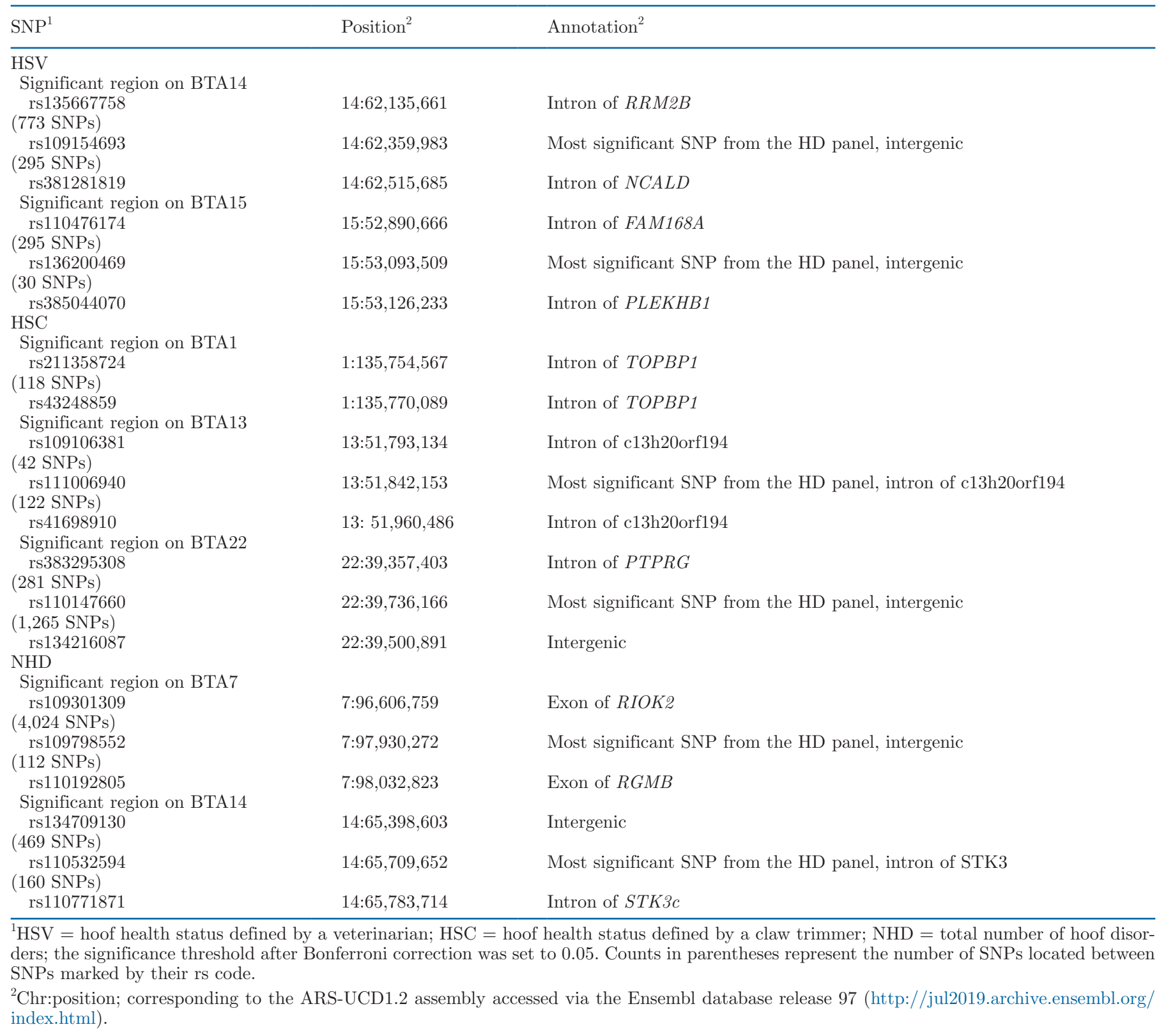

Table 3. Estimated variance components ( \pm standard error)

\begin{tabular}{lccccc}
\hline & & \multicolumn{2}{c}{ Variance component } & \\
\cline { 2 - 6 } Trait $^{1}$ & $\begin{array}{c}\text { Additive } \\
\text { polygenic }\end{array}$ & $\begin{array}{c}\text { Permanent } \\
\text { environmental }\end{array}$ & $\begin{array}{c}\text { Veterinarian/claw } \\
\text { trimmer }\end{array}$ & Residual & Heritability \\
\hline HSV & $0.002 \pm 0.001$ & $0.002 \pm 0.002$ & $10^{-6} \pm 10^{-6}$ & $0.044 \pm 0.002$ & $0.035 \pm 0.02$ \\
HSC & $0.075 \pm 0.014$ & $0.003 \pm 0.014$ & $0.061 \pm 0.026$ & $0.162 \pm 0.010$ & $0.249 \pm 0.04$ \\
NHD & $0.244 \pm 0.030$ & $10^{-7} \pm 10^{-8}$ & $0.168 \pm 0.072$ & $0.460 \pm 0.024$ & $0.279 \pm 0.03$ \\
\hline
\end{tabular}

${ }^{1} \mathrm{HSV}=$ hoof health status defined by a veterinarian; HSC $=$ hoof health status defined by a claw trimmer; NHD $=$ total number of hoof disorders. 


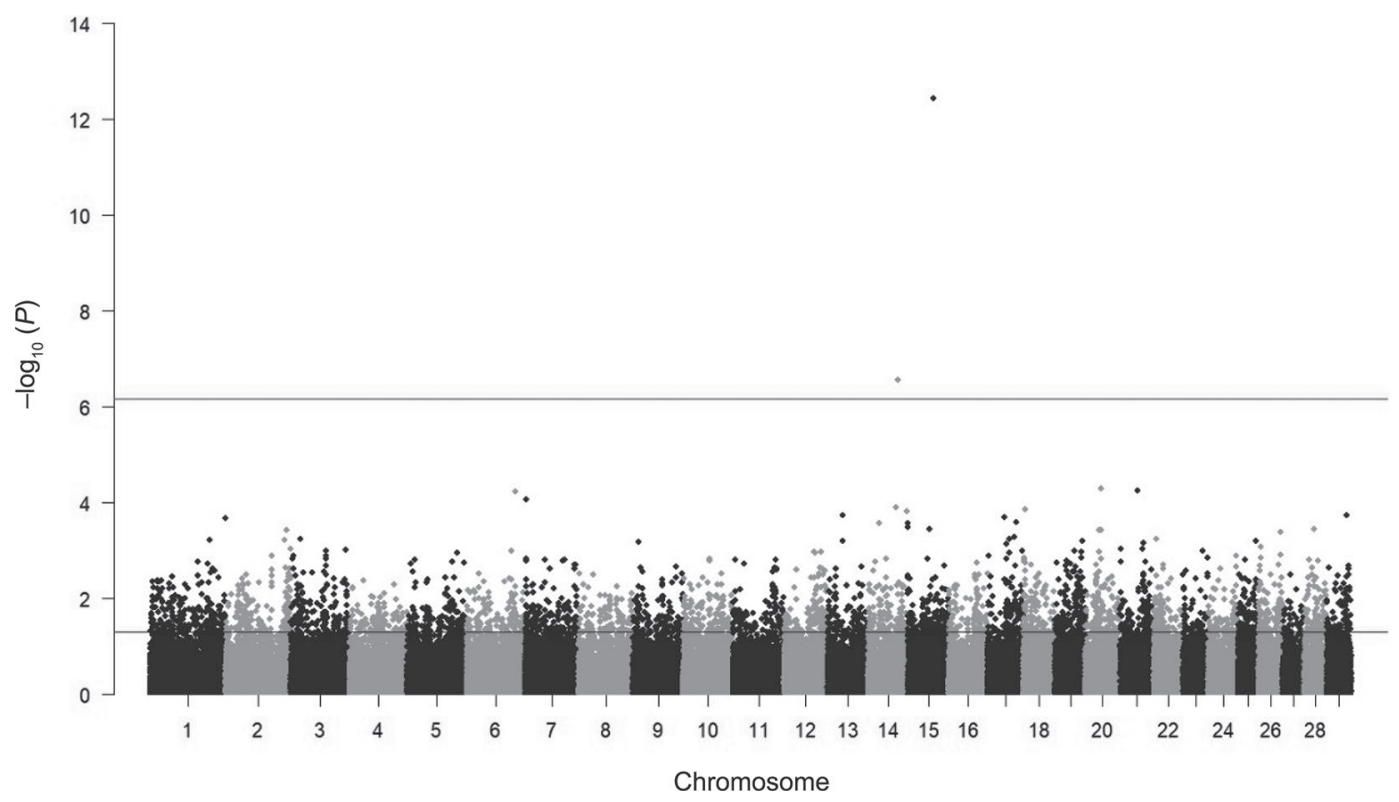

Figure 4. $P$-values for hoof health status defined by a veterinarian (HSV). The lower horizontal line corresponds to the - $\log _{10}$ of the nominal type I error probability of $5 \%$ and the upper horizontal line corresponds to the genome-wise $5 \%$ significance threshold after Bonferroni correction.

did not improve the resolution in detecting association signals because 1 of the 23 SNP was already identified in the first-stage GWAS.

Interesting results were obtained for HSC (Figure 8), albeit not within the significant region on BTA1, where, because of high inter-SNP LD, the same effect was estimated for all 120 SNP across the 15,522-bp region covering introns and exons of TOPBP1 (Table 2). On BTA13, where the significant region of 167,352 bp between rs109106381 and rs41698910 included 167 SNP, one of the most significant SNP (rs134142607) was located within an exon of C13H20orf194. On BTA22, the significant region comprised $143,488 \mathrm{bp}$, with 1,549 SNP flanked by rs383295308 and rs134216087.

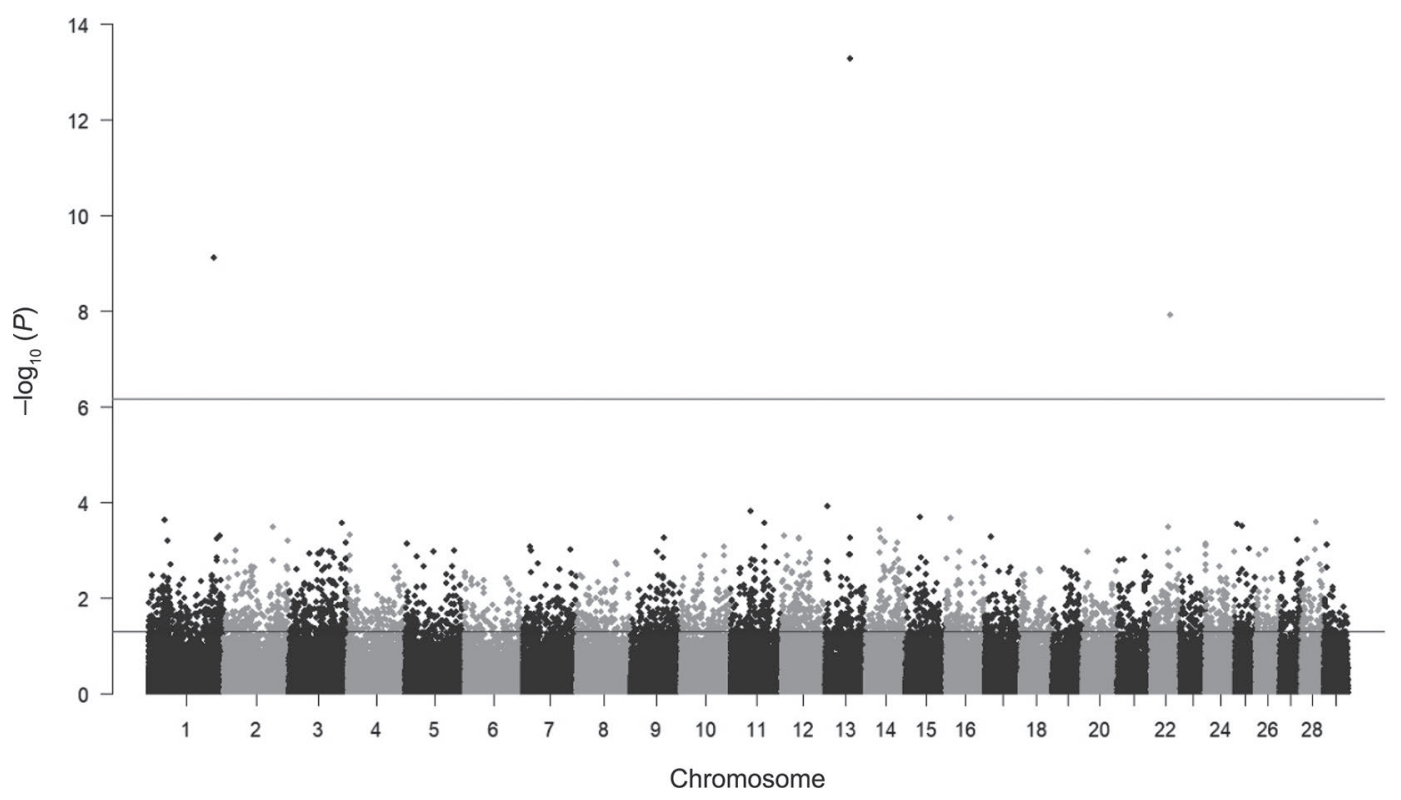

Figure 5. $P$-values for hoof health status defined by a claw trimmer (HSC). The lower horizontal line corresponds to the $-\log _{10}$ of the nominal type I error probability of $5 \%$ and the upper horizontal line corresponds to the genome-wise $5 \%$ significance threshold after Bonferroni correction. 


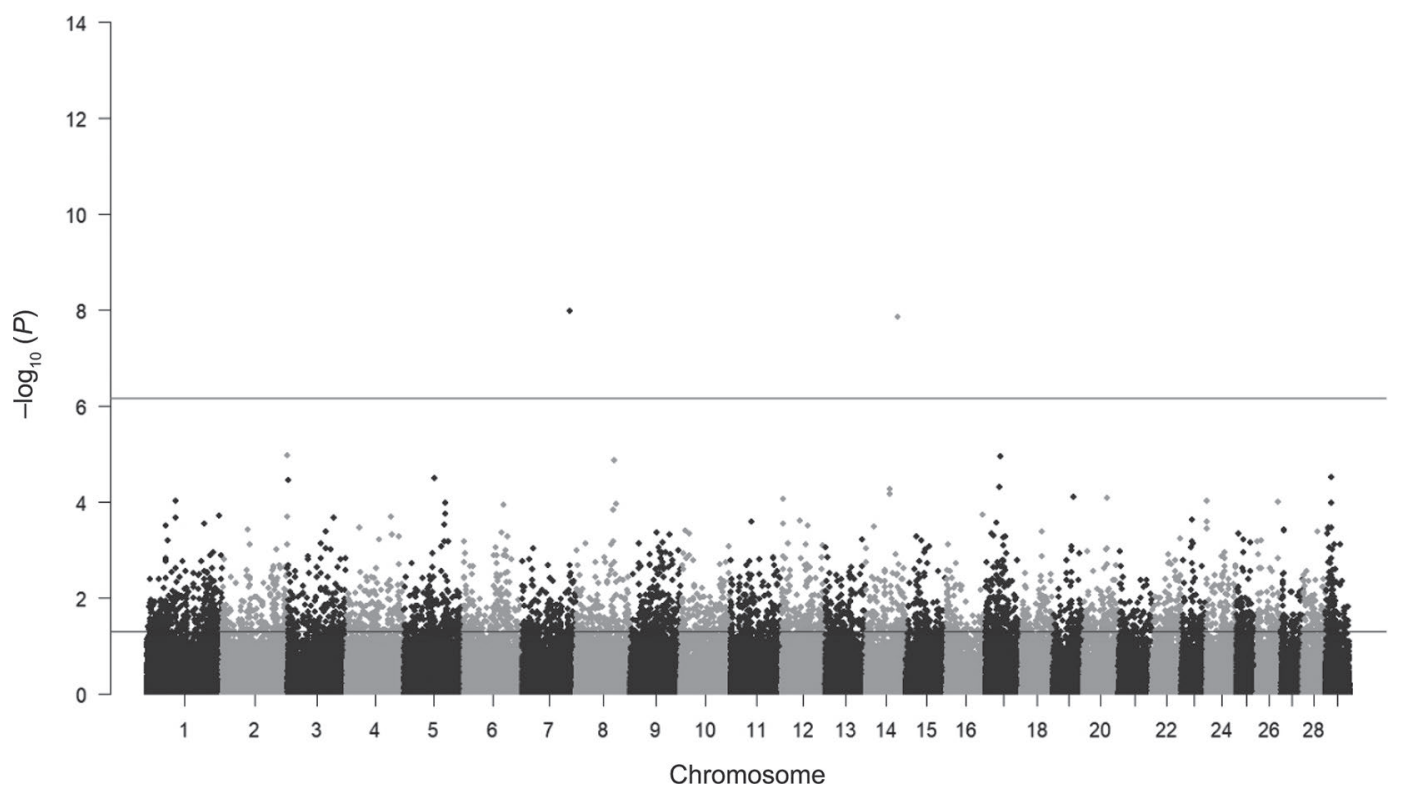

Figure 6. $P$-values for the total number of hoof disorders (NHD). The lower horizontal line corresponds to the - $\log _{10}$ of the nominal type I error probability of $5 \%$ and the upper horizontal line corresponds to the genome-wise $5 \%$ significance threshold after Bonferroni correction.

High SNP effects were located between 13:39,665,432 and $13: 39,697,735$, a region that includes rs 135082893 within an exon of a noncoding transcript sequence for the microRNA bta-mir-2369.

For NHD (Figure 9), 1 of 2 significant regions was detected on BTA7 between rs109301309 and rs110192805 (1,426,064 bp). The 34 most significant neighboring SNP were intergenic and contained the SNP that was the most significant in the first-stage GWAS. The genomic location of those SNP $(7: 97,891,749-7: 97,941,381)$ is relatively close to and downstream of an exon of
$R G M B$ gene (a distance of $91,238 \mathrm{bp}$ ) and an exon of a noncoding transcript of a long non-coding RNA (a distance of $65,299 \mathrm{bp}$ ). It also overlaps with a sequence corresponding to a computationally predicted gene. The second significant region was located on BTA14 between rs134709130 and rs110771871; it comprised 632 SNP spanning 385,111 bp downstream and within the $S T K 3$ gene. The most significant part of the region corresponded to an intron of STK3 and comprised 14 SNP, including the most significant SNP from the firststage GWAS.

Table 4. Significant SNP from the high-density panel

\begin{tabular}{llllr}
\hline SNP $^{1}$ & Position $^{2}$ & Effect & $\begin{array}{c}\text { Risk-increasing } \\
\text { allele }\end{array}$ & $P$-value $^{3}$ \\
\hline HSV & & & & \\
rs109154693 & $14: 62,359,983$ & 0.5793 & $\mathrm{C}$ & 0.0101 \\
rs136200469 & $15: 53,093,509$ & 0.8224 & $\mathrm{~A}$ & $<0.0001$ \\
HSC & $1: 135,826,020$ & 0.3459 & $\mathrm{~A}$ & $<0.0001$ \\
rs43247868 & $13: 51,842,153$ & 0.3206 & $\mathrm{G}$ & $<0.0001$ \\
rs111006940 & $22: 39,736,166$ & 0.3912 & $\mathrm{C}$ & 0.0003 \\
rs110147660 & $7: 97,930,272$ & & & 0.0005 \\
NHD & $14: 65,709,652$ & 0.1375 & $\mathrm{~A}$ & 0.0005 \\
rs109798552 & 0.1347 & $\mathrm{~A}$ & \\
rs110532594 & & & & \\
\hline
\end{tabular}

${ }^{1} \mathrm{HSV}=$ hoof health status defined by a veterinarian; HSC $=$ hoof health status defined by a claw trimmer; $\mathrm{NHD}=$ total number of hoof disorders. The significance threshold after Bonferroni correction was set to 0.05 .

${ }^{2}$ Chr:position; corresponding to the ARS-UCD1.2 assembly accessed via the Ensembl database release 97 (http: //jul2019.archive.ensembl.org/index.html).

${ }^{3}$ Corrected for multiple testing using the Bonferroni correction. 


\section{bta14}

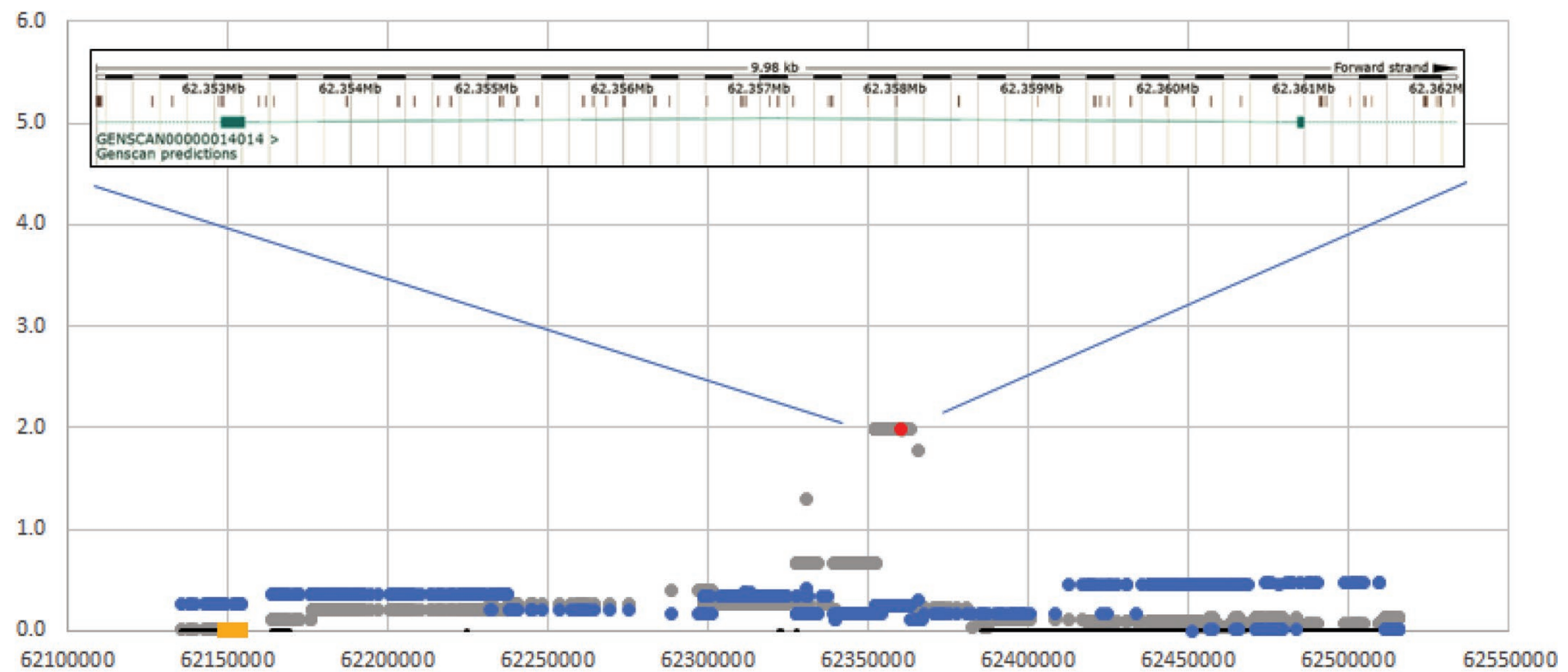

\section{bta15}

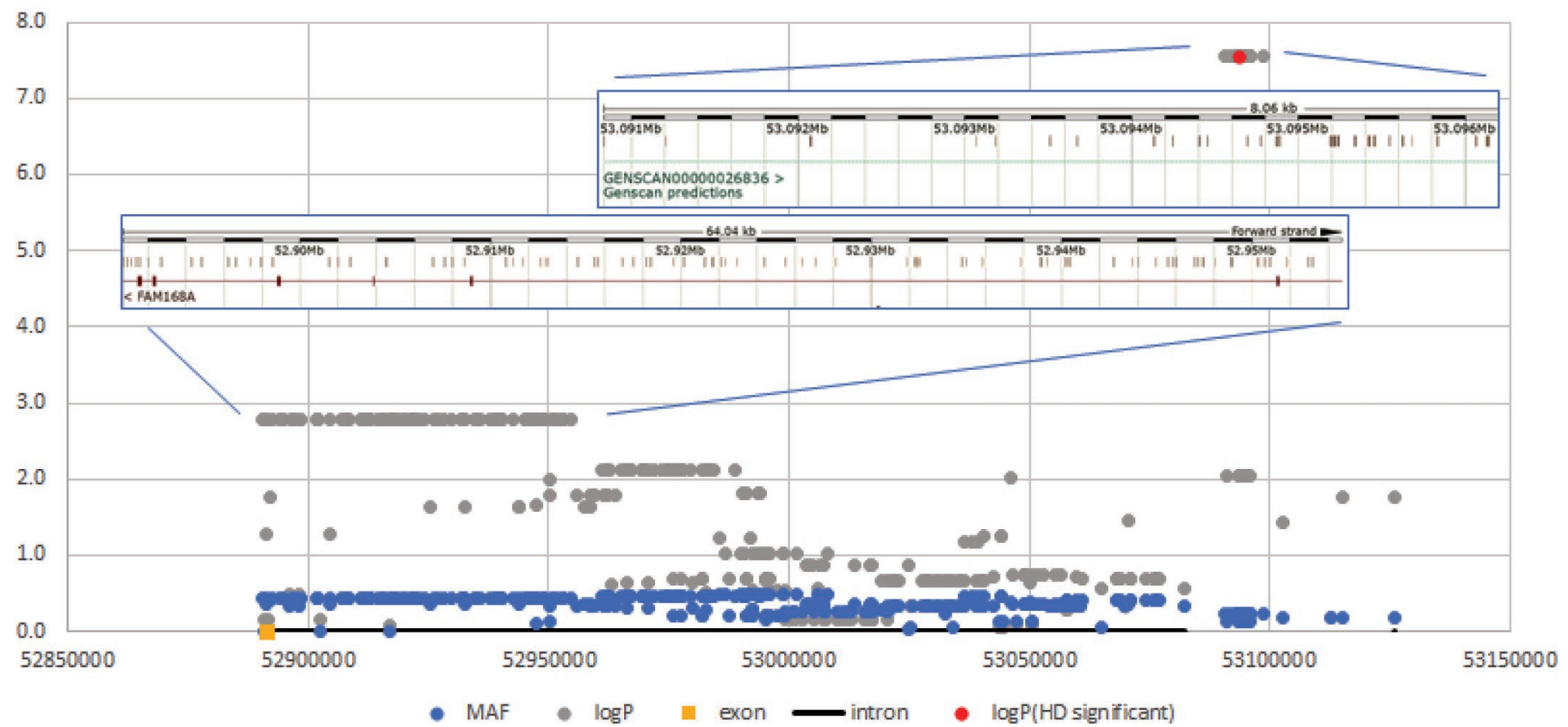

Figure 7. $P$-values and minor allele frequencies (MAF) for SNP within the regions significant for hoof health status defined by a veterinarian (HSV). The $\mathrm{x}$-axis represents SNP positions expressed in base pairs, the $\mathrm{y}$-axis represents $-\log _{10}(P)$, and the insets show the genomic context of the most significant SNPs on the ARS-UCD1.2 reference assembly (http://jul2019.archive.ensembl.org/index.html).

\section{DISCUSSION}

Heritabilities estimated in our study fell in the broad range of parameters reported previously and recently summarized by Heringstad et al. (2018). However, significant SNP locations showed no overlap between our study and published GWAS (van der Spek et al., 2015; Wu et al., 2016; Naderi et al., 2018; Croué et al., 2019). 
A similarly low concordance was observed by $\mathrm{Wu}$ et al. (2016), even within the same study and using different statistical models.

The relatively high heritabilities estimated in our study for HSC and NHD indicated good selection potential for reducing the susceptibility of hoof disorders as well as the possible involvement of candidate genes with medium to large effects. The low heritability of HSV indicated a very high environmental component, which was 1.7 times higher than for the similarly defined HSC. We hypothesize that the veterinary diagnosis is related to a much more severe hoof disorder stage, which involves the effect of many environmental factors, compared with a diagnosis made by claw trimmers on an earlier stage during routine hoof trimming, which often occurs at a subclinical stage of a disorder. Given the low heritability of this trait, no strong causal signals were expected.

One drawback of our study was the limited sample size, which influenced type I and type II error rates. This is also a possible explanation for the observed heterogeneity. In contrast, because hoof health traits are not subjected to routine recording in most countries, phenotyping is a limited factor in this and in other studies related to health traits. The training sample size available for imputation to WGS might have resulted in some imputation errors and did not permit good differentiation between closely linked SNPs in the second-stage GWAS. Within each significant region, groups of SNPs were in complete LD with the same estimated effects and thus significance. The lack of recombination events between those SNPs in the training data set did not allow for statistical differentiation among them. However, we identified genomic regions with significant effects on hoof health status and thus potential candidate genes located within or close to these regions: TOPBP1 (BTA1); RGMB (BTA7); C13H20orf194 (BTA13); NCALD, RRM2B, and STK3 (BTA14); FAM168A (BTA15); and PTPRG (BTA22). The most interesting findings indicate 2 possible causal mutations: rs135082893 within a sequence encoding a microRNA (bta-mir-2369) and rs134142607 within an exon of C13H20orf194 and in the STK3 gene, which is involved in a locomotion process as shown by its association with an "abnormal gait" phenotype in the mouse (http://www.mousephenotype.org).

\section{ACKNOWLEDGMENTS}

The research was supported by the Polish National Science Centre (NCN; grant no. 2015/19/B/ NZ9/03725), by the European Union's Seventh Framework Program for research, technological development and demonstration (under grant agreement 289592 266-Gene2Farm), and by the "Efficient Cow" project funded by the Austrian Federal Ministry of Agriculture, Forestry, Environment and Water Management, the Federations of Austrian Fleckvieh, Brown-Swiss, and Holstein, the Federation of Austrian Cattle Breeders and the Federal States of Austria. The authors have not stated any conflicts of interest.

Table 5. The most significant stage-two SNPs selected within the significant regions defined in the first-stage genome-wide association study

\begin{tabular}{|c|c|c|c|c|c|}
\hline Flanking SNP position (ID) ${ }^{1,2}$ & $\begin{array}{l}\text { No. of SNPs } \\
\text { in the region }\end{array}$ & $\begin{array}{l}\text { Region } \\
\text { length (bp) }\end{array}$ & Genomic annotation & Effect & $P$-value \\
\hline \multicolumn{6}{|l|}{ HSV } \\
\hline $\begin{array}{c}14: 62,352,133(\mathrm{rs} 41724166)- \\
62,363,182(\mathrm{rs} 136907591)\end{array}$ & 70 & 11,049 & $\begin{array}{l}\text { Within a predicted gene sequence, } \\
\text { downstream of } N C A L D\end{array}$ & 3.04 & 0.00240 \\
\hline $\begin{array}{c}15: 53,090,834(\mathrm{rs} 210116783)- \\
53,098,889(\mathrm{rs} 434361479)\end{array}$ & 23 & 8,055 & $\begin{array}{l}\text { Within a predicted gene sequence, } \\
\text { upstream of } F A M 168 A\end{array}$ & 4.30 & 0.00002 \\
\hline \multicolumn{6}{|l|}{ HSC } \\
\hline $\begin{array}{l}22: 39,665,432(\mathrm{rs} 137006000)- \\
39697630(\mathrm{rs} 133309559)\end{array}$ & 44 & 32,198 & $\begin{array}{l}\text { Within predicted gene sequence, within } \\
\text { noncoding bta-mir- } 2369 \text { upstream of } \\
P T P R G\end{array}$ & 4.03 & 0.00006 \\
\hline \multicolumn{6}{|c|}{ 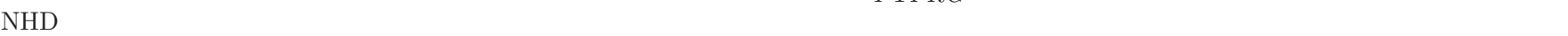 } \\
\hline $\begin{array}{l}\text { 7:97,891,749 (rs383780129)- } \\
97,941,381(\mathrm{rs} 136660809)\end{array}$ & 33 & 49,632 & $\begin{array}{l}\text { Within a predicted gene sequence, } \\
\text { upstream of } R I O K 2 \text {, downstream of } \\
R G M B\end{array}$ & 4.55 & 0.00001 \\
\hline
\end{tabular}

${ }^{1} \mathrm{HSV}=$ hoof health status defined by a veterinarian; HSC $=$ hoof health status defined by a claw trimmer; NHD $=$ total number of hoof disorders.

${ }^{2}$ Chr:position; corresponding to the ARS-UCD1.2 assembly accessed via the Ensembl database release 97 (http://jul2019.archive.ensembl.org/ index.html). 
bta01
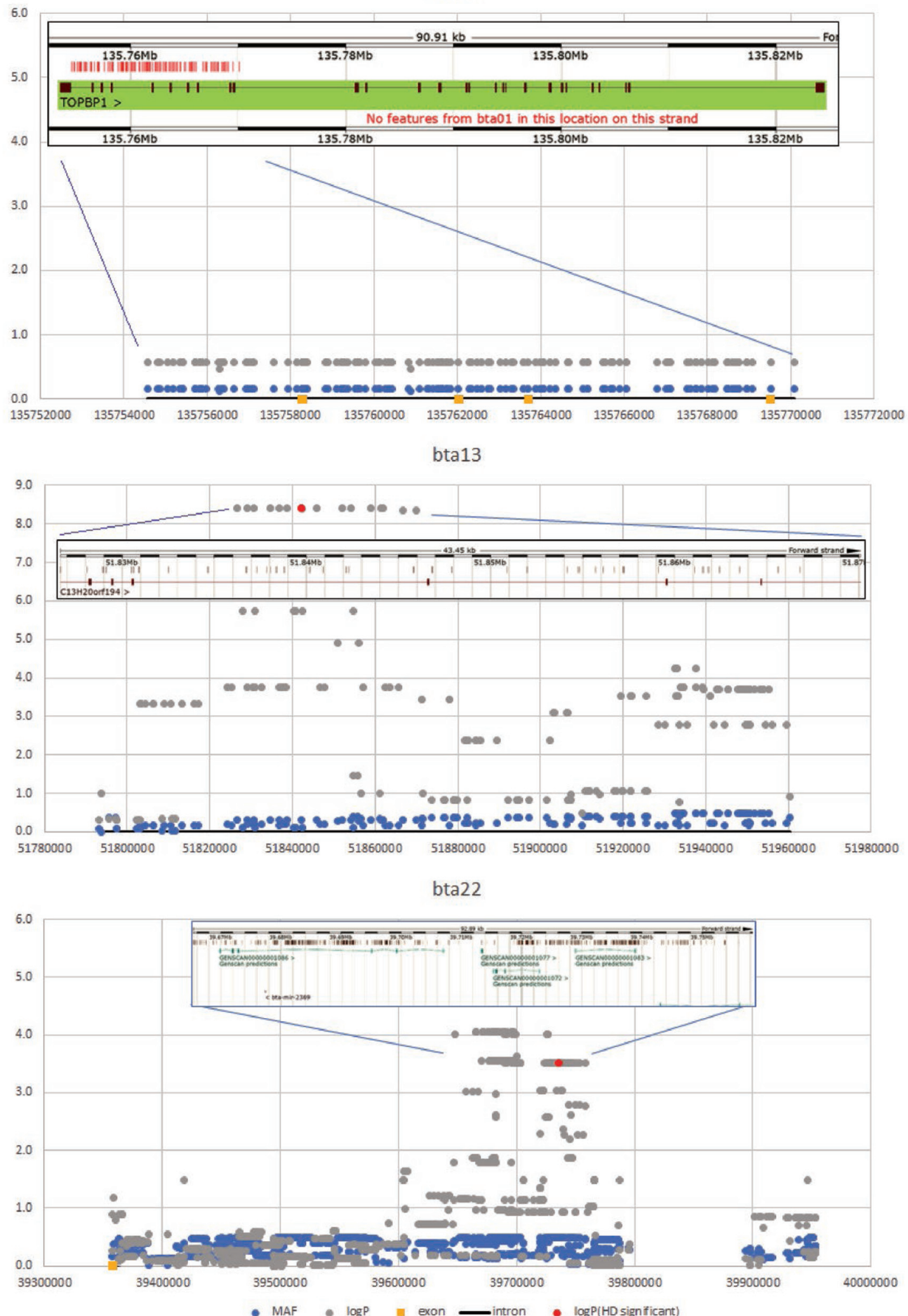

Figure 8. $P$-values and minor allele frequencies (MAF) for SNPs within the regions significant for hoof health status defined by a claw trimmer (HSC). The x-axis represents SNP positions expressed in base pairs, the $\mathrm{y}$-axis represents $-\log _{10}(P)$, and the insets show the genomic context of the most significant SNPs on the ARS-UCD1.2 reference assembly (http://jul2019.archive.ensembl.org/index.html). 
bta07
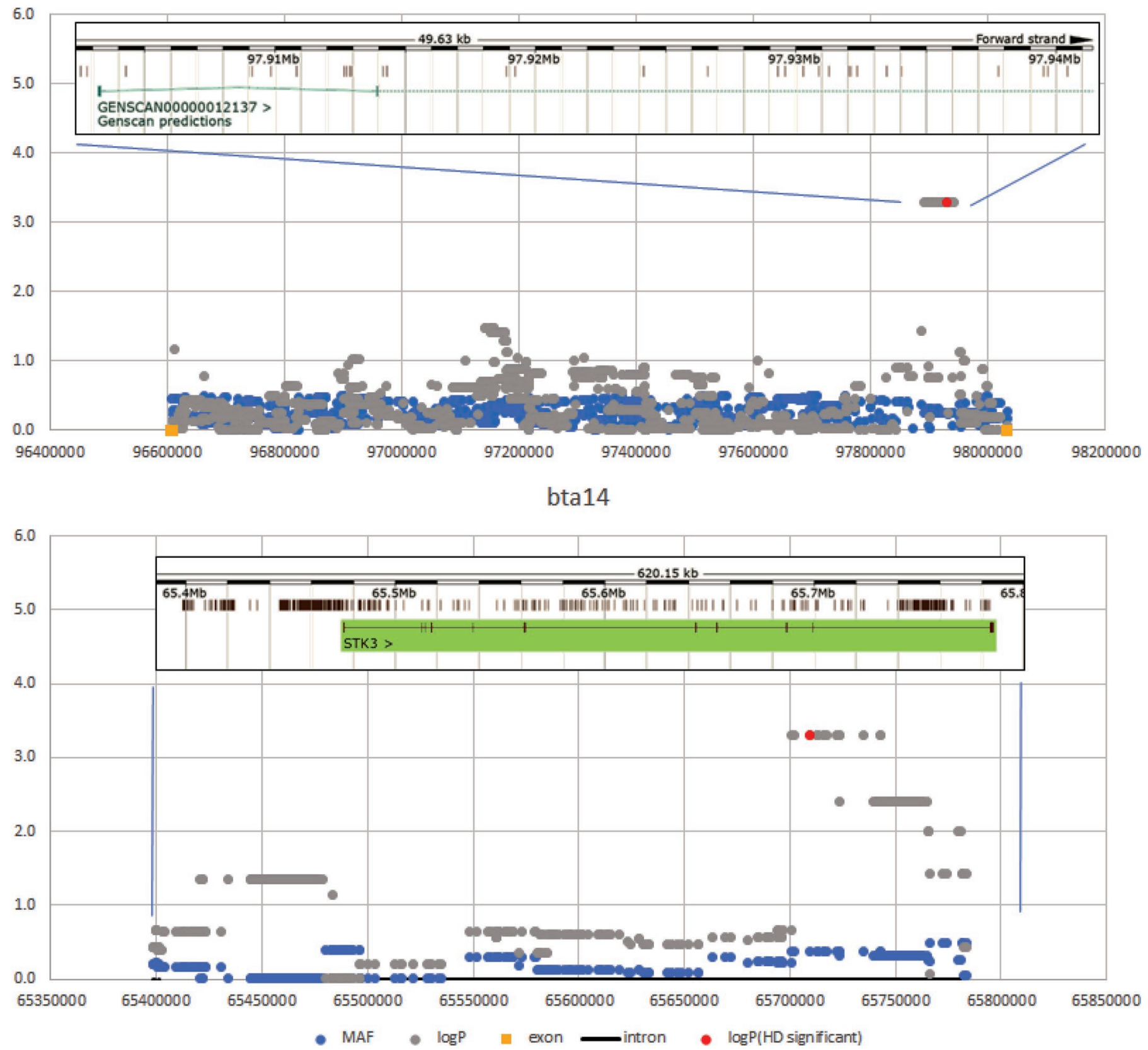

Figure 9. $P$-values and minor allele frequencies (MAF) for SNPs within the regions significant for the total number of hoof disorders (NHD). The $\mathrm{x}$-axis represents SNP positions expressed in base pairs, the y-axis represents $-\log _{10}(P)$, and the insets show the genomic context of the most significant SNPs on the ARS-UCD1.2 reference assembly (http://jul2019.archive.ensembl.org/index.html).

\section{REFERENCES}

Browning, S. R., and B. L. Browning. 2007. Rapid and accurate haplotype phasing and missing data inference for whole genome association studies by use of localized haplotype clustering. Am. J. Hum. Genet. 81:1084-1097. https://doi.org/10.1086/521987.

Buitenhuis, A. J., M. S. Lund, J. R. Thomasen, B. Thomsen, V. H. Nielsen, C. Bendixen, and B. Guldbrandtsen. 2007. Detection of quantitative trait loci affecting lameness and leg conformation traits in Danish Holstein cattle. J. Dairy Sci. 90:472-481. https:// doi.org/10.3168/jds.S0022-0302(07)72649-8.

Butler, D., B. R. Cullis, A. R. Gilmour, and B. J. Gogel. 2009. ASReml-R Reference Manual. Department of Primary Industries and Fisheries, Brisbane, QLD, Australia

Chapinal, N., A. Koeck, A. Sewalem, D. F. Kelton, S. Mason, G. Cramer, and F. Miglior. 2013. Genetic parameters for hoof lesions 
and their relationship with feet and leg traits in Canadian Holstein cows. J. Dairy Sci. 96:2596-2604. https://doi.org/10.3168/jds.2012 $-6071$.

Croué, I., F. Fikse, K. Johansson, E. Carlén, G. Thomas, H. Leclerc, and V. Ducrocq. 2017. Genetic evaluation of claw health traits accounting for potential preselection of cows to be trimmed. J. Dairy Sci. 100:8197-8204. https://doi.org/10.3168/jds.2017-13002.

Croué, I., A. Michenet, H. Leclerc, and V. Ducrocq. 2019. Genomic analysis of claw lesions in Holstein cows: Opportunities for genomic selection, quantitative trait locus detection, and gene identification. J. Dairy Sci. 102:6306-6318. https://doi.org/10.3168/ jds.2018-15979.

Dunnett, C. W. 1955. A multiple comparisons procedure for comparing several treatments with a control. J. Am. Stat. Assoc. 50:10961121. https://doi.org/10.1080/01621459.1955.10501294.

Hayes, B. J., and H. D. Daetwyler. 2019. 1000 Bull Genomes project to map simple and complex genetic traits in cattle: Applications and outcomes. Annu. Rev. Anim. Biosci. 7:89-102. https://doi.org/10 .1146/annurev-animal-020518-115024.

Heringstad, B., C. Egger-Danner, N. Charfeddine, J. E. Pryce, K. F. Stock, J. Kofler, A. M. Sogstad, M. Holzhauer, A. Fiedler, K. Müller, P. Nielsen, G. Thomas, N. Gengler, G. de Jong, C. Ødegård, F. Malchiodi, F. Miglior, M. Alsaaod, and J. B. Cole. 2018. Invited review: Genetics and claw health: Opportunities to enhance claw health by genetic selection. J. Dairy Sci. 101:4801-4821. https:// doi.org/10.3168/jds.2017-13531.

Li, J., and L. Ji. 2005. Adjusting multiple testing in multilocus analyses using the eigenvalues of a correlation matrix. Heredity 95:221227. https://doi.org/10.1038/sj.hdy.6800717.

Li, M. X., J. M. Yeung, S. S. Cherny, and P. C. Sham. 2012. Evaluating the effective numbers of independent tests and significant p-value thresholds in commercial genotyping arrays and public imputation reference datasets. Hum. Genet. 131:747-756. https://doi .org/10.1007/s00439-011-1118-2.

Malchiodi, F., A. Koeck, S. Mason, A. M. Christen, D. F. Kelton, F. S. Schenkel, and F. Miglior. 2017. Genetic parameters for hoof health traits estimated with linear and threshold models using alternative cohorts. J. Dairy Sci. 100:2828-2836. https://doi.org/10.3168/jds 2016-11558.

Naderi, S., M. Bohlouli, T. Yin, and S. König. 2018. Genomic breeding values, SNP effects and gene identification for disease traits in cow training sets. Anim. Genet. 49:178-192. https://doi.org/10 .1111 /age. 12661 .
Ødegård, C., M. Svendsen, and B. Heringstad. 2013. Genetic analyses of claw health in Norwegian Red cows. J. Dairy Sci. 96:7274-7283. https://doi.org/10.3168/jds.2012-6509.

Pérez-Cabal, M. A., and N. Charfeddine. 2015. Models for genetic evaluations of claw health traits in Spanish dairy cattle. J. Dairy Sci. 98:8186-8194. https://doi.org/10.3168/jds.2015-9562.

Ring, S. C., A. J. Twomey, N. Byrne, M. M. Kelleher, T. Pabiou, M. L. Doherty, and D. P. Berry. 2018. Genetic selection for hoof health traits and cow mobility scores can accelerate the rate of genetic gain in producer-scored lameness in dairy cows. J. Dairy Sci. 101:10034-10047. https://doi.org/10.3168/jds.2018-15009.

Swalve, H. H., H. Alkhoder, and R. Pijl. 2008. Estimates of breeding values for sires based on diagnoses recorded at hoof trimming: Relationships with EBV for conformation traits. Interbull Bull. 38:87-90.

Swalve, H. H., C. Floren, M. Wensch-Dorendorf, K. Schöpke, R. Pijl, K. Wimmers, and B. Brenig. 2014. A study based on records taken at time of hoof trimming reveals a strong association between the IQ motif-containing GTPase-activating protein 1 (IQGAP1) gene and sole hemorrhage in Holstein cattle. J. Dairy Sci. 97:507-519. https://doi.org/10.3168/jds.2013-6997.

van der Spek, D., J. A. M. van Arendonk, and H. Bovenhuis. 2015 Genome-wide association study for claw disorders and trimming status in dairy cattle. J. Dairy Sci. 98:1286-1295. https://doi.org/ 10.3168/jds.2014-8302.

van der Spek, D., J. A. M. van Arendonk, A. A. A. Vallee, and H. Bovenhuis. 2013. Genetic parameters for claw disorders and the effect of preselecting cows for trimming. J. Dairy Sci. 96:6070-6078. https://doi.org/10.3168/jds.2013-6833.

Weber, A., E. Stamer, W. Junge, and G. Thaller. 2013. Genetic parameters for lameness and claw and leg diseases in dairy cows. J. Dairy Sci. 96:3310-3318. https://doi.org/10.3168/jds.2012-6261.

Wu, X., B. Guldbrandtsen, M. S. Lund, and G. Sahana. 2016. Association analysis for feet and legs disorders with whole genome sequence variants in 3 dairy cattle breeds. J. Dairy Sci. 99:72217231. https://doi.org/10.3168/jds.2015-10705.

\section{ORCIDS}

T. Suchocki @ $\odot$ https://orcid.org/0000-0002-8530-3486

Ch. Egger-Danner ( ) https://orcid.org/0000-0002-8879-6845

J. Szyda @ https://orcid.org/0000-0001-9688-0193 